\title{
How may COVID-19 viral membrane resist when exposed radiation to treatment : effects on Phospholipids and cholesterol
}

Z. Khattari ( $\boldsymbol{D}$ zkhattari@hu.edu.jo)

Hashemite University

M. Sayyed

Isra University

\section{Research Article}

Keywords: SARS-COV-2, COVID-19, Cholesterol, viral Phospholipids Radiation resistance, Buildup factors

Posted Date: March 11th, 2021

DOI: https://doi.org/10.21203/rs.3.rs-276017/v1

License: (c) (i) This work is licensed under a Creative Commons Attribution 4.0 International License.

Read Full License 


\section{Abstract}

In this report, we have investigated the ability of the essential phospholipids and cholesterol found viral membrane of SARS-COV-2 to protect it from the radiation exposure. The applied mehod was Geometric Progression fitting procedure to obtained numerically the atomic number equivalenace $\left(\mathrm{Z}_{\text {eq }}\right)$, for any photon energy absorption exposure (EBF) and absorption (EABF) buildup factors. The used energy range $E \in[0.015,15] \mathrm{MeV}$ up to an ultimate penetration length of $40 \mathrm{mpf}$ for viral membrane essential components phospholipids and cholesterol. Most importantly, the calculated $\mathrm{Z}_{\text {eq }}$ values were found to vary with incident photon energy and phospholipids compositions. Also, both EBF and EABF were depend strongly on the photon energy and penetration depths. For phospholipids, these two factors almost coincide in the energy range studied while it slightly different for cholesterol. At energies $<150 \mathrm{keV}$, both buildup factors were found to to inversely proportional to $Z_{\text {eq }}$ values of the phospholipids and cholesterol. The buildup factors EABF and EBF were found to be larger for cholesterol than other phospholipids at nearly $60 \mathrm{keV}$ for COVID-19 viral membrane. The above results have shown that the viral membrane can resist the radiation effects and prevent the coronavirus form being inactivated when exposed to $Y$ - or $X$ rays radiotherapy treatment in medical trails except for a specific radiation energy depending on the $\mathrm{mfp}$ (i.e., $60 \mathrm{keV}$ at $5 \mathrm{mfp}$ ).

\section{Introduction}

Various techniques and methods have been emerged recently as prompt response to the COVID-19 treatment at different hospitals and research laboratories [1-5]. Among them is the use of irradiation ( $\gamma-$ rays), heat and UV treatment which in turn inactivate the virus effect by damaging its viral constitutes such as membrane surface RNA and DNA [1-7]. High doses of $Y$ - or X-rays in the range of several kGy (i.e., 3-7 kGy) for different viruses including the current COVID-19, caused by the severe acute respiratory syndrome coronavirus (SARS-COV-2). The later was declared in March 2019 by the WHO as a globe pandemic. Since the early discovery of the disease around 100 millions confirmed cases have been infected and more 2 millions have passed away according to WHO recent reports at the time of writing this report. Sine high doses of ${ }^{60} \mathrm{Co} Y$ - or X-ray can raise a destruction and loss to the lung interfacial molecules this has limit the use of this technique. On the other hand, low-energy electrons (200 keV) have replaced it for viral inactivation [4]. Such low-energy electron beam was sufficient to stop viral growth and keep antigenic properties of the human cell membrane lung [4]. Also, Corry et al. [2] has proposed that antioxidant Nacetyl-cysteine (NAC) could be used orally or within medical centers along with higher dose radiation in a clinical trial involved in COVID-19 [2]. The research group of Mehanna et al. has adviced the use of radiation therapy as an alternative to surgery for patients in various region of the world as a way to delay surgical operations which has been adapted rapidly in medical and radiation oncology [3].

In the past three decades, many research works have been extensively performed on various materials to study the absorption and transmission of $X$ and $y$-rays in various important biological samples [8-13]. These experimental and theoretical studies have been emerged because it is essential to imitate their 
functions in living cells. Most of these "in vitro" experiments have been performed in order to realize what is happing at cell level when X-or Y-ray interacts with tissues or cell membrane [14]. The photon energy ranged from $5 \mathrm{keV}$ to a few $\mathrm{MeV}$ is of great importance in medical, biological or agricultural applications [15]. However, for biologically-important elements of low $Z$ value, the attenuation coefficients measurement studies indicated that the radiations interact predominantly by the Compton effect and photoelectric effect in the energy range $E \in[0.2,1.5] \mathrm{MeV}$ (see [16] and the references therein). The tabulation of X-ray mass attenuation coefficients and interaction cross section coefficients for several elements, including biologically essential elements (i.e., $\mathrm{H}, \mathrm{C}, \mathrm{N}$ and $\mathrm{O}$ ) has been undergone several developments and updates and is now available as Web-based program accessible to all scientists around the globe. This version has also undergone further development to run under the Windows operating system platform which is now called WinXCom [22]. For the detailed and successive developments of the theoretical and experimental investigations of photon-matter interactions which have been implemented within this platform [16]. Also, from biological and medical viewpoint $y$-ray buildup factor is of great importance because of photon flux distribution and radiation dose received and buildup within the biologically essential macromolecules such as phospholipids, amino acids, peptides or proteins up to the cells or tissues. Of interest, the recognition how radiation-living matter interaction is cryptic in "buildup factors" [17]. Namely the exposure buildup factor (EBF) and the energy absorption buildup (EABF) factor. The factors are commently evaluated by Geometric Progression fitting procedure $[17,18]$.

In the following we summarize previous successful contributions which based on the buildup factors that have been carried out from simple chemical elements to more complex biological macromolecules relevant in human health and medicine. The early development of calculating the mass attenuation coefficients or photon interaction cross-sections for simple elements is dated back to the seminal work of Berge and Hubbell [19]. Since then there have been extensive theoretical and experimental research works to determine the buildup factors for complex biological macromolecules such as fatty acids, oils, carbohydrates, polypeptides or proteins and animal or plant tissues which is primarily composed of the life-essential elements $\mathrm{H}, \mathrm{C}, \mathrm{N}$ and $\mathrm{O}$ in varying proportions. Dried samples containing these essential elements have been reported by El-Kateb and Abdul-hamid [20] in the energy $E \in[54,1333] \mathrm{keV}$. Then one decade latter Sandhu et al. [21] have investigated a single-chain fatty acids almost in the same energy range. Then, a large body of publications have appeared in literature which is due to the WinXCom software development. This developed-software has allowed Gowda et al. [22] successfully measured the total attenuation and cross-sections for some sugar and amino acid macromolecules. Also, Manohara and Hanagodimth in couples of independent research reports have determined the effective electron densities and atomic numbers of certain types of amino acids in a broad range of photon energies from a few $\mathrm{keV}$ up to $100 \mathrm{GeV}$. A progressive investigation have also been done by Kurudirek et al. [23] who reported on the influence of gamma-ray radiation on human tissues by evaluating EBF and EABF. Moreover, the same research group, Kurudirek and Ozdemir [17] have effort the $y$-ray EABF) and EBF for different biological-related molecules when the energy $0.015<E<15 \mathrm{MeV}$ for various $\mathrm{mpf}$ values. In these inclusive works they have employed the five parameter G-P fitting method to deduct both EABF and EBF 
for amino acids, single-chain fatty acids and carbohydrates. On the other hand, Kore and Pawar [6] have measured the effective atomic number $\left(\mathrm{Z}_{\text {eff }}\right)$, electron density $\left(\mathrm{N}_{\text {eff }}\right)$ and mass attenuation coefficients of another class of amino acids which are also essential for the human body.

In the current research report work, we have focused on viral phospholipids and cholesterol the radiation effects and visus inactivation whn exposed to radiation. These molecules are in principle different from the previously investigated single-chain fatty acids with their acyl carbon double bond chains which span the cell membrane. The cell membrane is primarily a complex structure composed of phospholipid, cholesterol molecules and proteins where each of them has its own essential role or function within the cell in higher eukaryotic organisms [25]. The organization of phospholipids into two leaflets (i.e., membrane) in eukaryote cell membrane with a typical separation of $40 \mathrm{~nm}$ is vital for cell-interior protection from impaired radiations $[9,26]$. It is well established that the viral membrane separate the inner viral genetic materials from external surroundings. Many essentail cell living pathways accumalated at the outer viral membrane which usually prevents the whole cell from external stimuli. One of these sever stimulates is the external radiation effects exerted on the cell either by nature or in the case of medical treatments. In fact, the first barrier $\mathrm{X}$-rays and $\mathrm{y}$-rays radiations encounter to penetrate into cellinterior, causing a genetic damage in its compartments (e.g., DNA and RNA) is believed to be the cell's outer-leaflet monolayer. The phospholipids investigated in this study are zwitterionic (i.e., neutral) amphiphilic molecules with saturated double bond, containing 12-20 carbon atoms along their hydrophobic chains as depicted in Scheme 1. The headgroups of these lipid molecules possess a dipole moment with zero net charge. This choice of neutral phospholipids is meant to minimize the lipid's headgroups charge effects in the buildup factors calculations performed in this work. On the other hand, cholesterol which influences the conformation order, integrity or heterogeneity of the phospholipids carbon tails and membrane viscosity [27]. it is well known that biological membranes encapsules about 30-50 mol\% of cholesterol. Furthermore, cholesterol regulate the membrane bilayer thickness due to membrane-proteins interactions $[26,27]$. The molecular structure of the chosen phospholipids exemplified by DMPC and cholesterol is depicted in Scheme 1. The molecular formulas of the phospholipids and cholesterol used in the current report are listed in Table 1 (see supplementary material).

\section{Theoretical Methods Used For G-p Fitting Procdure}

The theoretical and computational work of buildup factor values and the G-P fitting parametersis fully detailed in the Supplemantry materials. The numerical calualctions used in this reprot are divided into three main sections. The first section is concerned for computation of the $Z_{\text {eq }}$ values for the lipids and cholesterol used in this study. The second one shows the calculations performed to obtainthe G-P fitting parameters and EBF and EABF numerical calculations are explanied in section three.

\section{Results And Discussion}


The molecular formula and composition of the lipids studied in this report is depicted in Table 1 (see supplementary material). The composition of each phospholipid will be correlated for each lipid with the two buildup factors (i.e., EBF and EABF) in the following discussion of the results. The dependency of equivalent atomic numbers $\left(Z_{\text {eq }}\right)$ as a function of the photon incident energy for DPPC and cholesterol is listed in the supplementary material (see Table 2 \& Table 3 ) respectively. The $Z_{\text {eq }}$ for other lipids are included in the supplementary material. Table $1 \&$ Table 2 present the equivalent atomic numbers and the associated absorption and exposure fitting parameters for the each lipid in the photon energy range $0.015<E<15 \mathrm{MeV}$. Noticeably, at a fixed photon incident energy the minimum and maximum for equivalent atomic numbers is appeared is for Cholesterol and DLPC respectively. This $Z_{\text {eq }}$ variation between the lipids can be correlated with the number of carbon atoms presented in each compound. It is clear from Table 1 (see supplementary material) that the only atomic weight fraction between the lipids constituent which may satisfy this relation is the C-atom. Other atomic weight fraction can't satisfy the behavior of the observed Zeq for the lipid used in this study. The EABF \& EBF) for each lipid is shown in Fig. 1 and Fig. 2 respectively as a function of $\gamma$ - or X-ray incident photon energy with different mean free path (i.e., $m f p \in[1,40]$ ) as specified within each panel. Comparison between different lipids EABP and EBF various photon energy at defined $\mathrm{mfp}$ values for these two important parameters has been depicted for all lipids graphically in Fig. 3 and Fig. 4. Effect due to lipid composition on them as a function of mean free path at fixed incident photon energy values is presented in Fig. 5 and Fig. 6 (see supplementary material). Finally, for the seek of comparison, we have presented a remarkable divergences between the EBF and EABF for two selected lipids namely, DLPC and cholesterol at fixed mfp values. These observations for $Z_{\text {eq }}, E A B F$ and EBF are discussed in the following sections.

\subsection{Gamma ray buildup factors of the phospholipids and cholesterol samples}

\subsubsection{Photon energy dependency}

Figure 1 and 2 give an inclusive representation about the EBF and EABF for each molecule reported here as a function photon of energy as indicated in the Figures. The largest values of these factors were obtained for cholesterol which posses at the same photon energy value a minimum of equivalent atomic numbers $\left(Z_{\text {eq }}\right)$, while the minimum values of EBF and EABF were obtained for DLPC which posses maximum of equivalent atomic numbers $\left(Z_{\text {eq }}\right)$ at the same photon energy. The Figures shows that $E B F$ and EABF regognized by having a quasi-Loretzian function with left-skewed behavoiur indicating the domination of the lower that higher photon energy-living matter interactions. It is also notoiced that the peak moves to right when the mfp values is increasing. This is a clear clue that one of the three wellknown scattering processes rule out the dominatation the other processes (see below for discussions).

The variation of energy EBF and EABF versus incident photon energy at selected mfp values for the phospholipids and cholesterol is depicted in Fig. 3 and Fig. 4. It is clear from these two figures that the all phospholipids (except cholesterol) showed almost consistent and almost identical variations of EBF and 
EABF with photon energy in the continuous energy region based on the domination photon interaction process in the studied energy range. For the compounds studied here, the two buildup factors were found to depend on the photon energy-lipids or Cholesterol interactions in the studied energy zone. The EBF and EABF were found to have a small value in the lower $\gamma$ - or $\mathrm{X}$-ray energy zone than that at higher photon energy levels where it reaches a maximum peak at intermediate energy values. It worthy noticeable that, the buildup factors for phospholipids and cholesterol were match in the higher photon energy zone and diverge in the lower photon energy zone keep both of them larger for cholesterol than other phospholipids. These noticed differences can be easily attributed by employing the three well-know photon scattering process when interacting with matter. The pair production processes and photoelectric phenomenon manifest for a short period of time in the low and high photon energy zone respectively, while Compton scattering process is pronounced at intermediate phone energy lasting for longer period of time resulting in huge buildup factors when compared with the other scattering processes. The last process can maximally enhance both buildup factors by multiple photons scattering inside the lipids or cholesterol elements. For example, Cholesterol and other phospholipids have the peaked EBF values at $0.06,0.08 \mathrm{MeV}$, while the peaked EABF values are at 0.05 and $.08 \mathrm{MeV}$, respectively at $1 \mathrm{mfp}$. These energy values were the largest value of the buildup factor appearing at higher energy values. These results showed that the two buildup factors were totally independent from phospholipid chemical compositions in the whole energy zone studied here except for cholesterol. From these two figures it is also appeared that the buildup factors attaining their peaked values at $40 \mathrm{mfp}$ which is the outliers for our numerical calculation carried out in this report. In fact, for $\mathrm{mfp}>40$ another mathematical and theoretical formalism must be adopted to gain insight about their variations with energy or mfp [21]. This observed behavior is true for all phospholipids investigated in this study expect cholesterol. In the studied energy range $Z_{\text {eq }} \in[5,7]$ for phospholipids while for cholesterol it is always lower than these values at fixed incident photon energy (i.e., < 5.6). Practically, these effect can be understood with the aid of the fact that smaller buildup factors may be correlated with entire atomic structure of for these phospholipids with higher values of equivalent atomic numbers (i.e., $\mathrm{Z}_{\mathrm{eq}}>5.6$ ).

\subsubsection{Penetration depth dependency}

The dependency of EABF and EBF with penetration depth at specific values of incident photon energy is shown in Fig. 5 and Fig. 6 (see supplementary material) respectively. Both Figures showing that at low photon energy, Cholesterol has the greatest values among other lipids since it has low equivalent atomic numbers $Z_{\text {eq }}$ value when compared with other lipids. Other phospholipids almost follow the same trend with no significance difference in both buildup factors. These observations ensure that effect such as penetration depth can be overcome by tuning the photon energy to achieve a specific goal in practical use especially in medicinal applications. It worth also nothing that this phenomenon can be correlated with the figures also showed that at $\mathrm{E}=0.15 \mathrm{MeV}$, the buildup factors reaches their peaked value in the mean free path lengths studied here. The above observation can be discussed in terms of the chemical composition of the phospholipids and cholesterol presented in this report. This observed similarity in the buildup factors is due to the fact that phospholipids almost having the same chemical structural compositions with minor variations in their molecular weight fractional percentage. These molecules 
possess the same molecular structure with hydrophobic headgroups and hydrophilic tails spanning the cell membrane (see Scheme 1), then these behavior was expected, on the other hand cholesterol is different in composition and structure with a rigid molecular ring composed of tetra-atomic molecular system with a hydrogen double bonding between the $\mathrm{C}$-atoms with hydroxyl group which provides the molecule with its amphiphilic integrity. Our data clearly demonstrate that the molecular structure of the studied lipids is crucial for the $y$ - or $X$ ray radtion EBF and EABF in living-matter.

\subsection{Comparison between EABF and EBF}

Figure 7 shows the relative differences (\%) between EABF and EBF. This methodology of numerical analysis may assist the occurrence in the maximum photon radiation intensity when interacting with matter. This is practically very useful when to judge whether this maximum intensity occurring inside or outside the cell. The relative difference alternates between both buildup factors indicating which one is dominate at a particular photon energy and/or mfp. Also, it is clear that the maximum difference exists in the intermediate energy zone where Compton scattering phenomenon is the primary process among other processes. This process, in turn, leads to huge peak for $\operatorname{EBF}$ and $\operatorname{EABF}[13,17,23]$. Figure $7 \mathrm{a}$ and Fig. $7 \mathrm{~b}$ (see supplementary material) showing these calculated two factors for DLPC and Cholesterol with maximum and minimum $\mathrm{Z}_{\mathrm{eq}}$ respectively among all the investigated compounds. Maximum differences were obtained at $88 \%$ and $65 \%$ for cholesterol and DLPC respectively. This observed relative differences can be explained by their low or high equivalent atomic numbers.

\section{Conclusions}

In this report, the results calculated about the equivalent atomic numbers for different acyl chain length phospholipids and cholesterol. The $\gamma$-ray EBF and EABF have been calculated for this life-essential molecules in energy range $E \in 0.015<E<15 \mathrm{MeV}$ for different values of $40 \mathrm{mfp}$. Our results shows that the viral membrane contiguous can protect the interior viral and genetic material of coronaviruses in the whole range of the of the energy range studied expect for a certain values of the radiation energy at specific $\mathrm{mfp}$. For example at $\mathrm{mfp}=5$, the viral membrane can resist radiation and protect the virus being damaged in the whole energy range studied expect at $60 \mathrm{keV}$. These valuable results have also shown that the presence of these phospholipids in the outermost viral membrane plays a vital role for the cellinterior (i.e., DNA and RNA) protection.

\section{Declarations}

Consent for publication: The Authors of this article grants the Publisher the sole and exclusive license of the full copyright in the Contribution, which license the Publisher hereby accepts.

Availability of data and materials: The datasets supporting the conclusions of this article are included within the article (and its additional files).

Funding: There is no funding. 
Conflict of Interest: Ziad Khattari declares that he has no conflict of interest. Mohammad Sayyed declares that he has no conflict of interest.

Ethical approval: This article does not contain any studies with human participants or animals performed by any of the authors.

\section{References}

1. Maro Durante, Kai Schults, S. Incerti, Z. Francis, S. Zein, C. A. Guzman, Virus irradiation and COVID-19 Disease, Forntiers in Physics:8, 565861.

2. Corry, Peter M., and Griffin, Robert J., A Radiation Mitigator as a Potential Treatment for COVID-19, Radiation Research, 193(6): 505-507.

3. Hisham Mehanna, Maura Gillison, Anne W.M. Lee, Sandra V. von Zeidler, and Sandro Porced, Adapting Head and Neck Cancer Management in the Time of COVID-19, Int. J Radiat Oncol Biol Phys 2020;107:628-630.

4. Fertey J., Bayer, L. Grunwald, T. Pohl A., Beckmann J., Gotzmann G., et al., Pathogens inactivated by low-energy-electron irradiation maintain antigetic properties and induced protictive immune response. Viruses (2016) 8:319

5. George D. Wilson, Minesh P. Mehta, James S. Welsh, Arnab Chakravarti, C. Leland Rogerse and James Fontanesia, Investigating Low-Dose Thoracic Radiation as a Treatment for COVID-19 Patients to Prevent Respiratory Failure, RADIATION RESEARCH 194, 1-8 (2020)

6. Friederike Feldmann, W. Lesley Shupert, Elaine Haddock, Barri Twardoski, and Heinz Feldmann, Gamma Irradiation as an Effective Method for Inactivation of Emerging Viral Pathogens, Am. J. Trop. Med. Hyg., 100(5), 2019, pp. 1275-1277

7. Pataje G. Prasanna, Gayle E. Woloschak, Andrea L. DiCarlo, Jeffrey C. Buchsbaum et al., Low-Dose Radiation Therapy (LDRT) for COVID-19: Benefits or Risks?, RADIATION RESEARCH 194, 452-464 (2020)

8. L. Gerward et al., X-ray absorption in matter re-engineering XCOM. Radiat. Phys. Chem. 60, 23-24 (2001); L. Gerward, N. Guilbert, K.B. Jensen, and H. Levring, Win Xcom- a programee for calculating Xray attenuation coefficients. Radiat. Phys. Chem. 71, 53-54 (2004)

9. A.B. Chilton, J.K. Shultis, and R. Faw, "Principle of Radiation Shielding," 1st ed. (Prentic-Halle, Englewood Cliffs, New Jersey, 1984).

10. G.S. Bhandal, and K. Singh, "Effective atomic number studies in different biological samples for partial and total photon interactions in the energy range $10^{-3}$ to $10^{5} \mathrm{MeV}$," Int. J. Appl. Radiat. Isot. 44, 505-510 (1997).

11. S.R. Manohara, and S.M. Hanagodimath, "Studies on effective atomic numbers and electron densities of essential amino acids in the energy range $1 \mathrm{keV}-100 \mathrm{GeV}$," Ncul. Instrum. Methods Phys. Res. B 258, 321-328 (2007). 
12. S.R. Manohara, S.M. Hanagodimath, and L. Gerward, "Energy dependenece of effective atomic numbers for photon energy absorption and photon interaction: Studies of some biological molecules in the energy range $1 \mathrm{keV}-20 \mathrm{MeV}$," Med. Phys. 52, 388-402 (2008).

13. P.S. Kore, and P.P Pawar, "Measurements of mass attenuation coefficient, effective atomic number and electron density of some amino acids," Radiat. Phys. Chem. 98, 86-91 (2014).

14. ANSI/ANS-6.4.3, Gamma ray attenuation coefficient and buildup factors ffor engineering materials, American Nuclear Society, La Grange Park, Illinois, 1991.

15. J.H. Hubbell, "Review of photon interaction cross section data in medical and biological context," Phys. Med. Biol. 44, R1-R22 (1993).

16. J.H. Hubbell, "Summary of existing information on the incoherent scattering of photons, particularly on validity of the use of incoherent scattering function," Radiat. Phys. Chem. 50, 113-124 (1997).

17. M. Kurudirek, and Y. Özdemir, "A comprehensive study on energy absorption and exposure buildup factors for some essential amino acids and carbohydrates in the energy range $0.0015-15 \mathrm{MeV}$ up to 40 mean free path," Nucl. Instrum. Methods Phys. Res. B 269, 7-19 (2011).

18. Y. Harima, Y. Sakamoto, S. Tanaka, and M. Kawai, "Validity of the geometric-progression formula in approximation of gamma ray buildup factors," Nucl. Sci. Eng. 94, 24-(1986).

19. M.J. Berg, and J.H. Hubbell, XCOM: Photon Cross Section Data base. Web Version 1.2, National Institute of Standards and Technology, Gaithersburg, MD 20899, USA, August 1999, Originally published at NBSIR 87-3597, XCOM: Photon Cross Section on a Personal Computer (July 1987), 1987-1999, 19990 Available from: http://physics.nist.gov./xcom.

20. A.H. El-Kateb, A.S. Abul Hamid, "Photon attenuation coefficient study of some materials containing hydrogen, carbon and oxgen," Appl. Radiat. Isot. 42, 303-307 (1991).

21. G.K. Sandhu, K. Singh, B.S. Lark, and L. Gerward, "Molar extinction coefficients of some fatty acis," Radiat. Phys. Chem. 65, 211-215 (2002).

22. S. Gowda, S. Krishnaveni, and R. Gowda, "Studies on effective atomic numbers and electron densities in amino acids and sugurs in energy range 30-1333 keV," Ncul. Instrum. Methods Phys. Res. B 239, 361-369 (2005).

23. M. Kurudirek, B. Dogan, M. Ingec, N. Ekinci, and Y. Özdemir, "Gamma-ray energy absorption and exposure buildup factor studies in some human tissues with endometriosis," Appl. Radiat. Isot. 69, 382-388 (2011).

24. M. Kurudirek, and Y. Özdemir, "Gamma-ray energy absorption and exposure buildup factor studies in some human tissues with endometriosis," Ncul. Instrum. Methods Phys. Res. B 239, 361-369 (2011).

25. R.B. Gennis, "Biomembranes Molecular Structure and Function," 1st ed., (Springer-Verlag, New York, 1988).

26. K. Simons, and E. Ikonen, " Functional rafts in cell membranes," Nature, 387, 569-572 (1997).

27. E. London, "Insights into Lipid Domain/Raft Structure and Formation from Experiments in Model Membrane," Curr. Opn. Str. Bio. 12, 480-486 (2002). 
28. Y. Sakamoto, and D.K. Trubey, "Radiation safty information computation center data package DLC129/ANS643, Geometric Prog. Gamma-ray Buildup Factor Coefficients,".

\section{Tables}

Table 1 Chemical formulas and compositions of the investigated lipids in weight fraction.

\begin{tabular}{llccccc}
\hline \hline Lipid & Chemical formula & $\mathrm{H}$ & $\mathrm{O}$ & $\mathrm{C}$ & $\mathrm{N}$ & $\mathrm{P}$ \\
\hline DAPC & $\mathrm{C}_{48} \mathrm{H}_{96} \mathrm{NO}_{8} \mathrm{P}$ & 0.114340 & 0.151247 & 0.681261 & 0.016551 & 0.036601 \\
\hline DOPC & $\mathrm{C}_{44} \mathrm{H}_{84} \mathrm{NO}_{8} \mathrm{P}$ & 0.107701 & 0.162818 & 0.672263 & 0.017817 & 0.039400 \\
\hline DPPC & $\mathrm{C}_{40} \mathrm{H}_{80} \mathrm{NO}_{8} \mathrm{P}$ & 0.109850 & 0.174368 & 0.654505 & 0.019081 & 0.042196 \\
\hline DMPC & $\mathrm{C}_{36} \mathrm{H}_{72} \mathrm{NO}_{8} \mathrm{P}$ & 0.107047 & 0.188799 & 0.637805 & 0.020661 & 0.045688 \\
\hline DLPC & $\mathrm{C}_{32} \mathrm{H}_{64} \mathrm{NO}_{8} \mathrm{P}$ & 0.103738 & 0.205834 & 0.618092 & 0.022525 & 0.049810 \\
\hline Cholesterol & $\mathrm{C}_{27} \mathrm{H}_{46} \mathrm{O}$ & 0.119912 & 0.041378 & 0.838710 & 0 & 0 \\
\hline \hline
\end{tabular}

DAPC: 1,2-diarachidonoyl-sn-glycero-3-phosphocholine

DOPC: 1, 2-dioleoyl-sn-glycero-3-phosphocholine

DPPC: 1, 2-dipalmitoyl-sn-glycero-3-phosphocholine

DMPC: 1,2, dimyristoyl-sn-glycero-3-phosphocholine

DLPC: 1,2, dimyristoyl-sn-glycero-3-phosphocholine

Table 2 Equivalent atomic number $\left(\mathrm{Z}_{\mathrm{eq}}\right)$ and G-P exposure (EBF) and energy absorption (EABF) buildup factor coefficients for cholesterol. 


\begin{tabular}{|c|c|c|c|c|c|c|c|c|c|c|c|}
\hline \multirow{2}{*}{$\begin{array}{l}\text { Energy } \\
(\mathrm{MeV})\end{array}$} & \multirow[t]{2}{*}{$\mathrm{Z}_{\mathrm{eq}}$} & \multicolumn{5}{|c|}{ EBF } & \multicolumn{5}{|c|}{ EABF } \\
\hline & & b & c & $\mathrm{a}$ & $\mathrm{X}_{\mathrm{k}}$ & d & b & c & $\mathrm{a}$ & $\mathrm{x}_{\mathrm{k}}$ & d \\
\hline 0.015 & 5.689 & 1.486 & 0.581 & 0.131 & 14.518 & -0.064 & 1.502 & 0.570 & 0.137 & 14.595 & -0.069 \\
\hline 0.02 & 5.698 & 2.108 & 0.796 & 0.063 & 15.767 & -0.033 & 2.125 & 0.800 & 0.061 & 16.074 & -0.033 \\
\hline 0.03 & 5.704 & 3.841 & 1.295 & -0.052 & 13.281 & 0.020 & 4.002 & 1.285 & -0.050 & 13.203 & 0.019 \\
\hline 0.04 & 5.706 & 5.713 & 1.882 & -0.144 & 14.103 & 0.063 & 5.200 & 1.868 & -0.142 & 14.163 & 0.062 \\
\hline 0.05 & 5.715 & 7.117 & 2.255 & -0.184 & 14.375 & 0.080 & 5.629 & 2.179 & -0.175 & 14.552 & 0.073 \\
\hline 0.06 & 5.724 & 7.563 & 2.528 & -0.211 & 14.486 & 0.093 & 5.402 & 2.385 & -0.194 & 14.706 & 0.081 \\
\hline 0.08 & 5.709 & 7.126 & 2.822 & -0.237 & 14.500 & 0.103 & 4.781 & 2.571 & -0.210 & 14.859 & 0.084 \\
\hline 0.1 & 5.767 & 6.406 & 2.852 & -0.239 & 14.759 & 0.103 & 4.247 & 2.599 & -0.212 & 14.989 & 0.083 \\
\hline 0.15 & 4.641 & 5.622 & 3.434 & -0.281 & 15.062 & 0.117 & 3.502 & 2.487 & -0.192 & 17.892 & 0.056 \\
\hline 0.2 & 4.828 & 4.001 & 3.325 & -0.286 & 14.187 & 0.126 & 3.229 & 2.148 & -0.184 & 17.880 & 0.062 \\
\hline 0.3 & 4.000 & 3.448 & 3.442 & -0.304 & 14.200 & 0.142 & 2.722 & 1.060 & -0.175 & 15.880 & 0.059 \\
\hline 0.4 & 5.000 & 2.862 & 2.630 & -0.245 & 12.580 & 0.111 & 2.533 & 2.027 & -0.167 & 14.340 & 0.065 \\
\hline 0.5 & 5.000 & 2.679 & 2.375 & -0.222 & 12.680 & 0.104 & 2.430 & 1.860 & -0.148 & 14.530 & 0.061 \\
\hline 0.6 & 5.000 & 2.547 & 2.161 & -0.198 & 13.690 & 0.102 & 2.358 & 1.720 & -0.129 & 14.730 & 0.051 \\
\hline 0.8 & 8.763 & 2.166 & 1.500 & -0.096 & 14.509 & 0.038 & 2.218 & 1.505 & -0.097 & 14.517 & 0.038 \\
\hline 1 & 8.000 & 2.098 & 1.418 & -0.084 & 14.350 & 0.033 & 2.104 & 1.427 & -0.086 & 14.200 & 0.035 \\
\hline 1.5 & 4.789 & 2.090 & 1.406 & -0.091 & 13.629 & 0.053 & 1.935 & 1.287 & -0.063 & 14.203 & 0.028 \\
\hline 2 & 4.969 & 1.958 & 1.215 & -0.051 & 14.408 & 0.028 & 1.833 & 1.183 & -0.042 & 14.121 & 0.019 \\
\hline 3 & 4.945 & 1.789 & 1.070 & -0.018 & 14.388 & 0.011 & 1.715 & 1.054 & -0.013 & 12.924 & 0.005 \\
\hline 4 & 5.009 & 1.684 & 0.978 & 0.006 & 14.190 & -0.005 & 1.630 & 0.986 & 0.004 & 14.938 & -0.003 \\
\hline 5 & 5.018 & 1.599 & 0.933 & 0.018 & 14.941 & -0.010 & 1.571 & 0.938 & 0.017 & 13.854 & -0.010 \\
\hline 6 & 5.018 & 1.541 & 0.904 & 0.026 & 14.909 & -0.014 & 1.525 & 0.903 & 0.027 & 13.154 & -0.014 \\
\hline 8 & 5.007 & 1.447 & 0.873 & 0.035 & 14.183 & -0.020 & 1.451 & 0.856 & 0.043 & 11.158 & -0.023 \\
\hline 10 & 5.006 & 1.383 & 0.853 & 0.041 & 13.422 & -0.018 & 1.389 & 0.856 & 0.040 & 14.509 & -0.021 \\
\hline 15 & 4.988 & 1.286 & 0.830 & 0.048 & 13.984 & -0.024 & 1.295 & 0.834 & 0.047 & 14.026 & -0.026 \\
\hline
\end{tabular}

Table 3 Equivalent atomic number $\left(\mathrm{Z}_{\mathrm{eq}}\right)$ and $\mathrm{G}-\mathrm{P}$ exposure $(\mathrm{EBF})$ and energy absorption (EABF) buildup factor coefficients for DPPC. 


\begin{tabular}{|c|c|c|c|c|c|c|c|c|c|c|c|}
\hline \multirow{2}{*}{$\begin{array}{l}\text { Energy } \\
(\mathrm{MeV})\end{array}$} & \multirow[t]{2}{*}{$Z_{\text {eq }}$} & \multicolumn{5}{|c|}{ EBF } & \multicolumn{5}{|c|}{ EABF } \\
\hline & & b & c & $\mathrm{a}$ & $\mathrm{X}_{\mathrm{k}}$ & d & b & c & $\mathrm{a}$ & $\mathrm{x}_{\mathrm{k}}$ & d \\
\hline 0.015 & DPPC & 1.229 & 0.472 & 0.172 & 14.283 & -0.086 & 1.231 & 0.481 & 0.165 & 14.487 & -0.080 \\
\hline 0.02 & 7.055 & 1.518 & 0.575 & 0.134 & 14.954 & -0.066 & 1.533 & 0.568 & 0.138 & 14.843 & -0.069 \\
\hline 0.03 & 7.125 & 2.497 & 0.815 & 0.060 & 15.854 & -0.041 & 2.594 & 0.802 & 0.066 & 15.065 & -0.039 \\
\hline 0.04 & 7.207 & 3.728 & 1.201 & -0.036 & 13.545 & 0.010 & 3.778 & 1.196 & -0.034 & 13.647 & 0.009 \\
\hline 0.05 & 7.256 & 4.753 & 1.542 & -0.096 & 13.759 & 0.040 & 4.681 & 1.537 & -0.095 & 13.799 & 0.039 \\
\hline 0.06 & 7.280 & 5.222 & 1.817 & -0.137 & 13.702 & 0.062 & 5.014 & 1.810 & -0.136 & 13.738 & 0.061 \\
\hline 0.08 & 7.305 & 5.182 & 2.131 & -0.176 & 13.465 & 0.080 & 4.925 & 2.105 & -0.172 & 13.538 & 0.077 \\
\hline 0.1 & 7.369 & 5.005 & 2.235 & -0.183 & 14.237 & 0.079 & 4.652 & 2.201 & -0.179 & 14.261 & 0.077 \\
\hline 0.15 & 7.270 & 3.938 & 2.321 & -0.193 & 14.237 & 0.084 & 3.808 & 2.237 & -0.182 & 14.407 & 0.074 \\
\hline 0.2 & 7.328 & 3.460 & 2.461 & -0.213 & 13.643 & 0.092 & 3.330 & 2.226 & -0.182 & 14.794 & 0.076 \\
\hline 0.3 & 6.805 & 3.147 & 2.413 & -0.211 & 14.410 & 0.094 & 2.797 & 2.129 & -0.175 & 14.960 & 0.069 \\
\hline 0.4 & 6.000 & 2.862 & 2.630 & -0.245 & 12.580 & 0.111 & 2.533 & 2.027 & -0.167 & 14.340 & 0.065 \\
\hline 0.5 & 5.000 & 2.487 & 1.743 & -0.131 & 14.187 & 0.051 & 2.476 & 1.753 & -0.133 & 14.251 & 0.053 \\
\hline 0.6 & 7.807 & 2.366 & 1.644 & -0.118 & 14.301 & 0.045 & 2.367 & 1.658 & -0.120 & 14.304 & 0.048 \\
\hline 0.8 & 7.904 & 2.241 & 1.580 & -0.112 & 14.030 & 0.048 & 2.202 & 1.544 & -0.105 & 14.200 & 0.043 \\
\hline 1 & 7.000 & 2.098 & 1.418 & -0.084 & 15.700 & 0.023 & 2.104 & 1.427 & -0.086 & 14.200 & 0.035 \\
\hline 1.5 & 8.000 & 2.087 & 1.400 & -0.089 & 13.656 & 0.052 & 1.935 & 1.287 & -0.062 & 14.229 & 0.028 \\
\hline 2 & 4.825 & 1.922 & 1.201 & -0.047 & 14.178 & 0.024 & 1.839 & 1.174 & -0.039 & 14.363 & 0.016 \\
\hline 3 & 5.561 & 1.762 & 1.061 & -0.015 & 12.199 & 0.007 & 1.715 & 1.051 & -0.012 & 14.056 & 0.003 \\
\hline 4 & 5.693 & 1.662 & 0.984 & 0.005 & 23.169 & -0.007 & 1.627 & 0.989 & 0.003 & 13.441 & -0.002 \\
\hline 5 & 5.691 & 1.582 & 0.937 & 0.017 & 14.627 & -0.011 & 1.566 & 0.944 & 0.015 & 14.707 & -0.008 \\
\hline 6 & 5.700 & 1.530 & 0.907 & 0.026 & 14.533 & -0.015 & 1.521 & 0.901 & 0.029 & 12.603 & -0.017 \\
\hline 8 & 5.671 & 1.442 & 0.870 & 0.037 & 16.191 & -0.031 & 1.436 & 0.875 & 0.036 & 11.818 & -0.018 \\
\hline 10 & 5.676 & 1.376 & 0.854 & 0.042 & 12.678 & -0.020 & 1.381 & 0.858 & 0.040 & 14.378 & -0.022 \\
\hline 15 & 5.677 & 1.279 & 0.838 & 0.047 & 14.886 & -0.028 & 1.287 & 0.837 & 0.047 & 15.347 & -0.031 \\
\hline
\end{tabular}

\section{Figures}




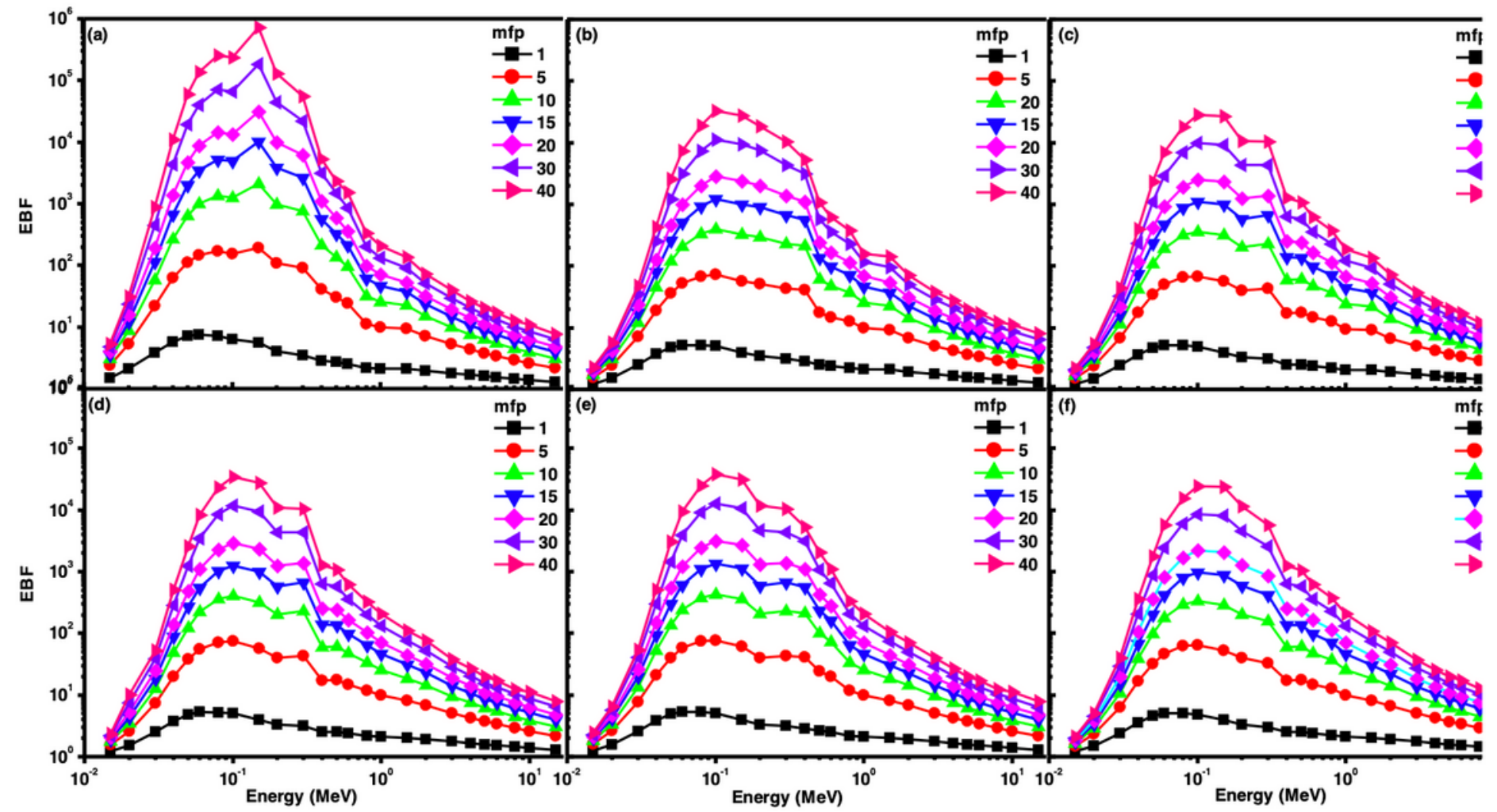

Figure 1

(a-f) Variation of exposure buildup factors with photon energy for (a) Cholesterol, (b) DPPC, (c) DMPC, (d) DOPC, (e) DAPC and (f) DLPC.

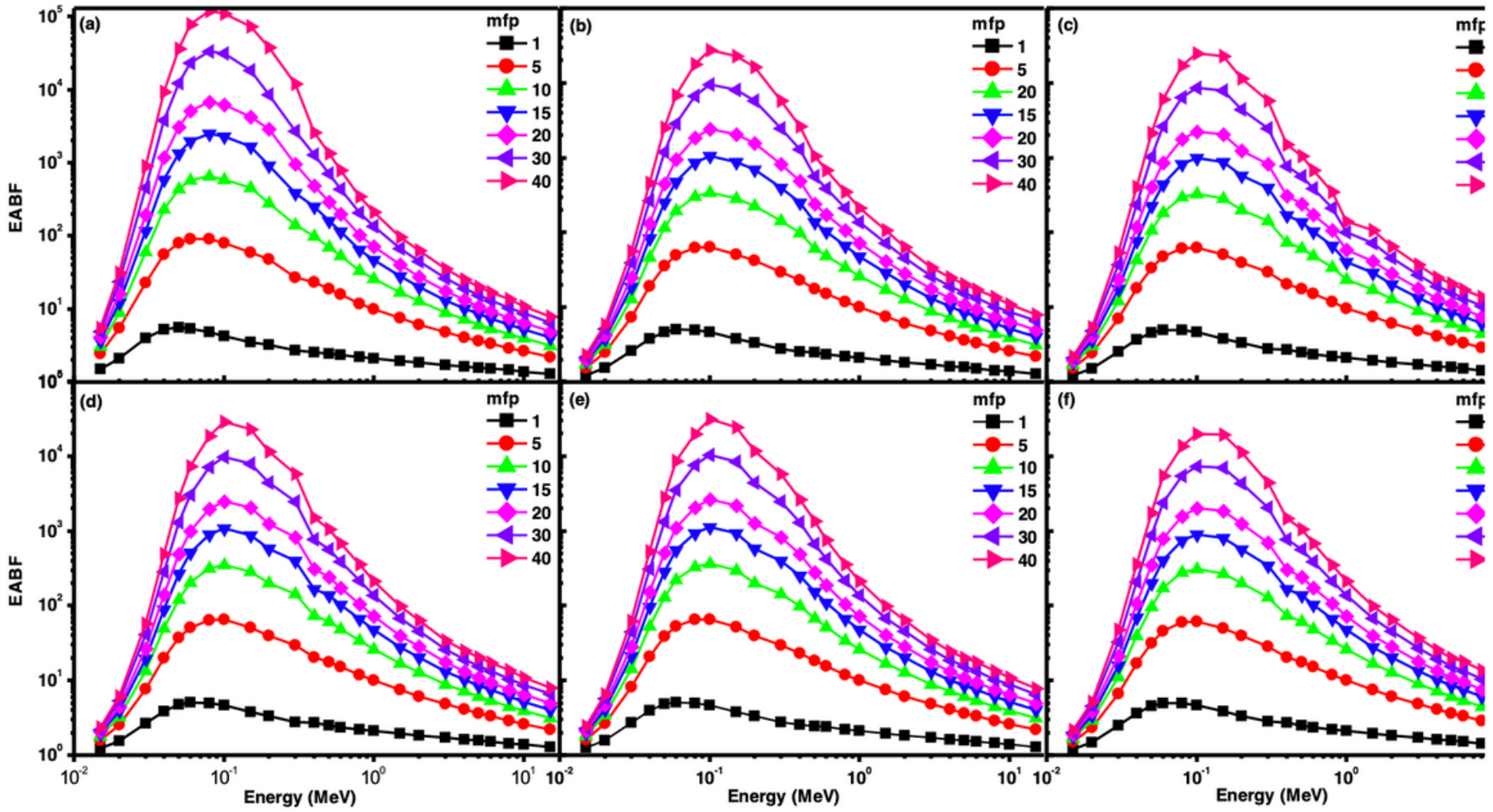


Figure 2

(a-d) Variation of energy absorption buildup factors with photon energy for (a) Cholesterol, (b) DPPC, (c) DMPC, (d) DOPC, (e) DAPC and (f) DLPC.
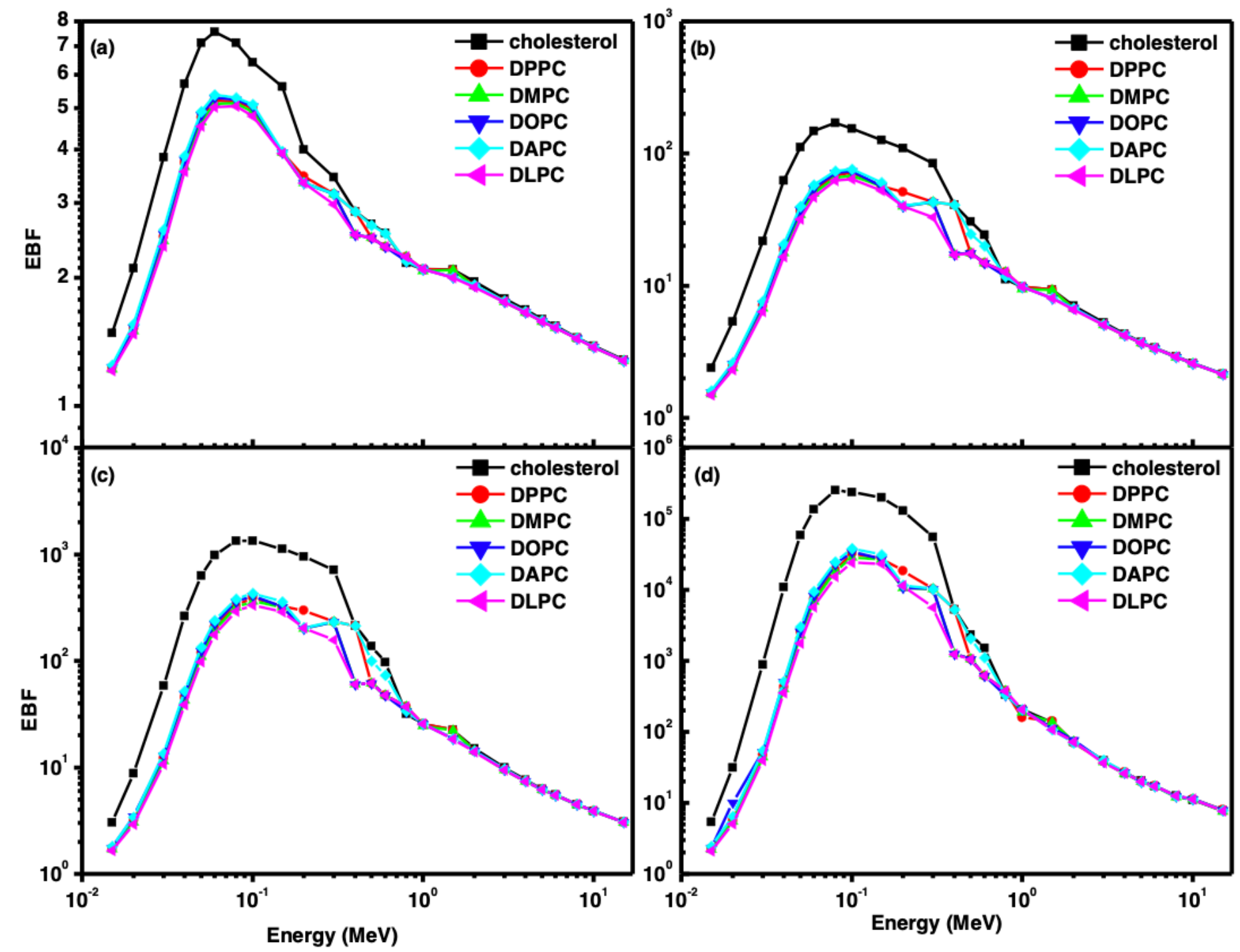

Figure 3

(a-d) Variation of exposure buildup factors with photon energy at (a) 1, (b) 5, (c) 10 and (d) $40 \mathrm{mfp}$. 


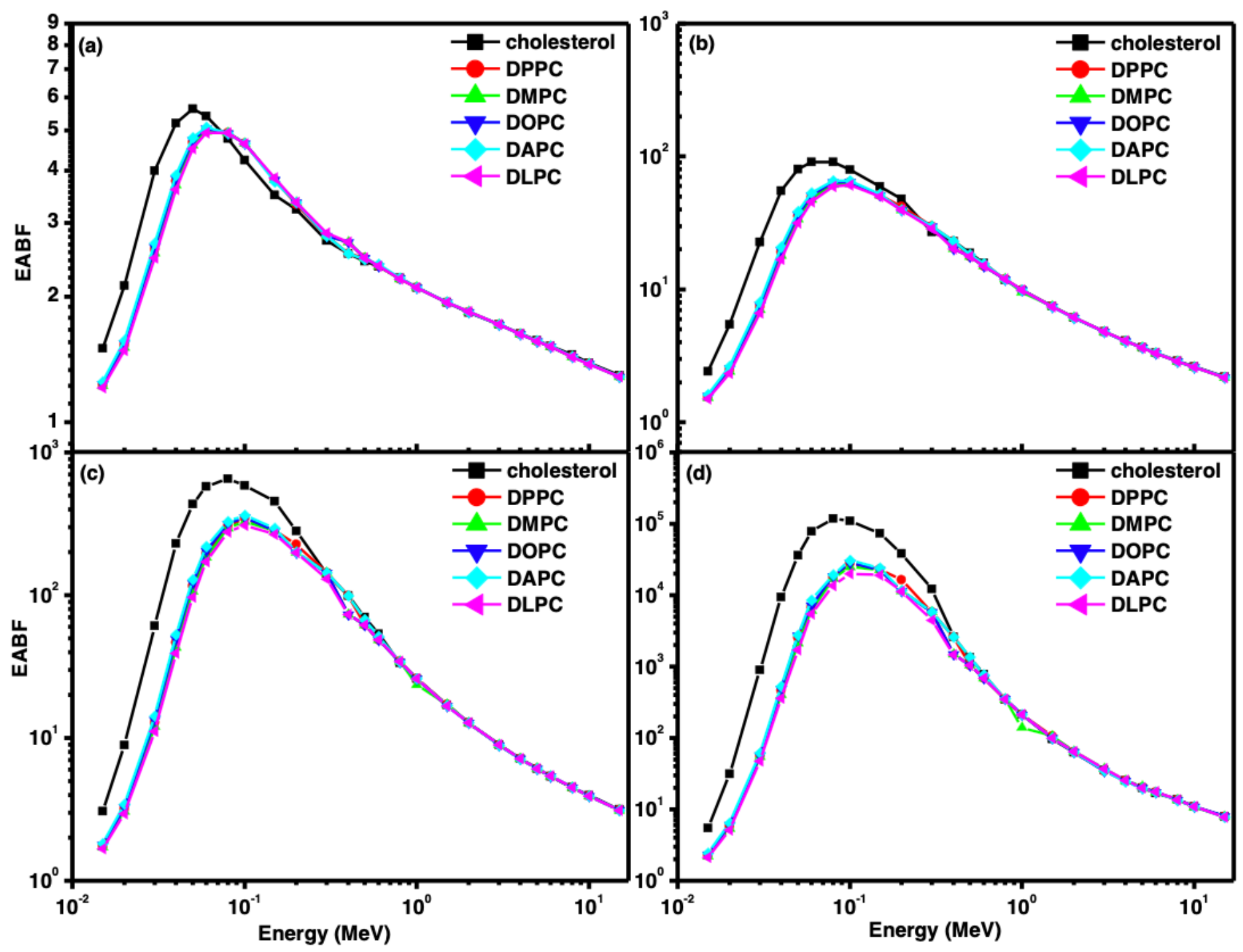

Figure 4

(a-d) Variation of energy absorption buildup factors with photon energy at (a) 1, (b) 5, (c) 10 and (d) 40 mfp. 

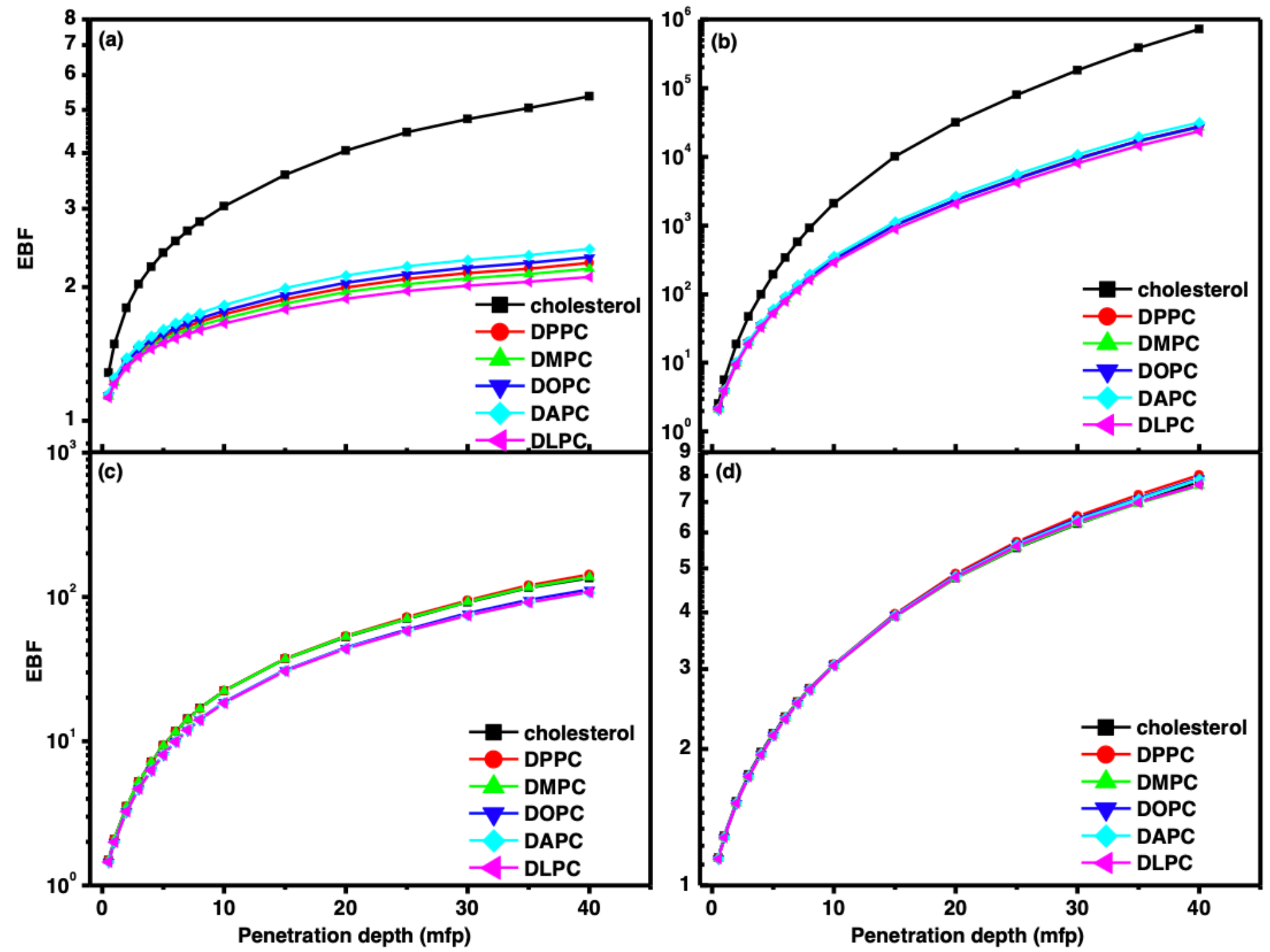

Figure 5

(a-d) Variation of exposure buildup factors with penetration depth (mpf) at (a) 0.015 , (b) 0.15 , (c) 1.5 and (d) $15 \mathrm{MeV}$. 

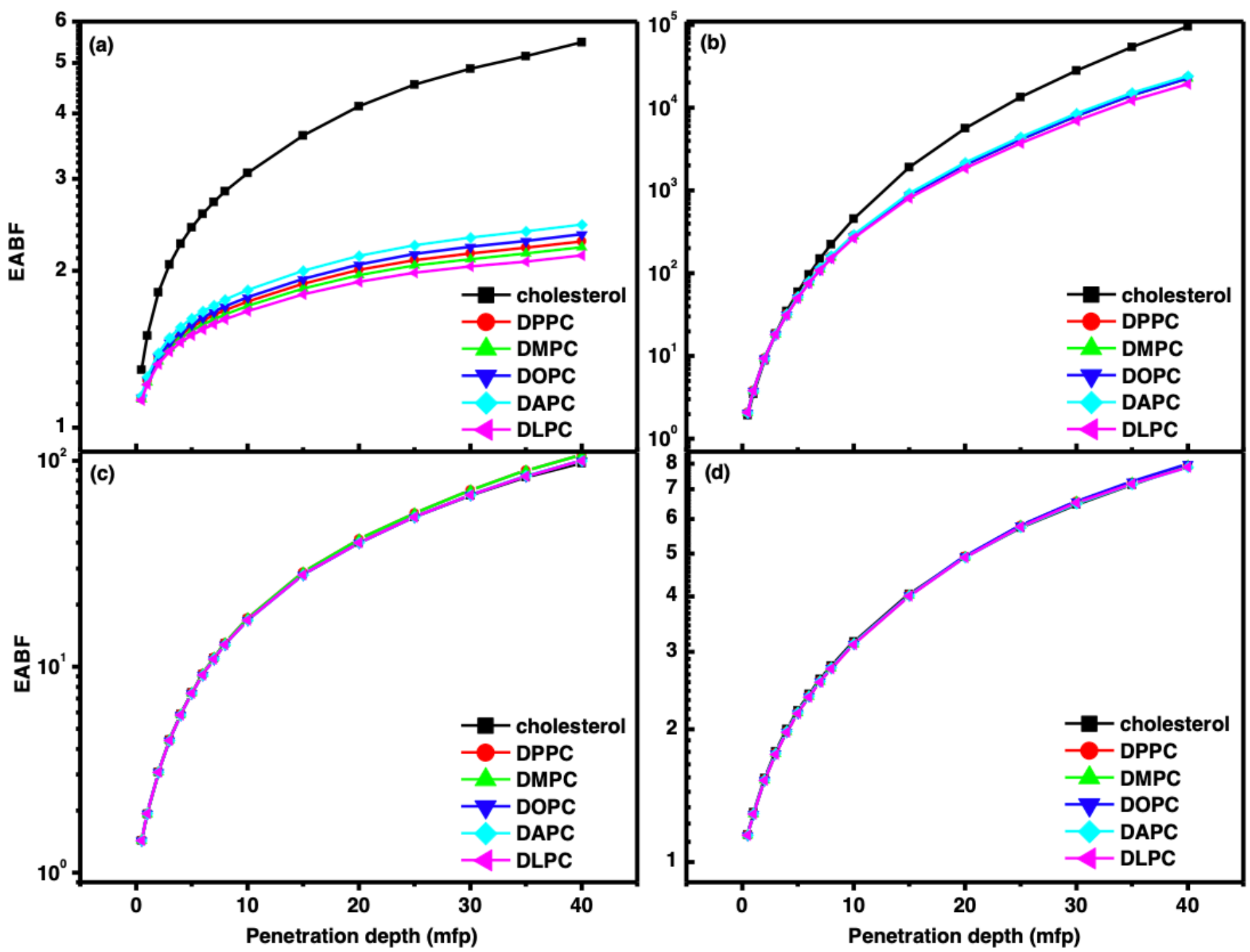

Figure 6

(a-d) Variation of energy absorption buildup factors with penetration depth (mpf) at (a) 0.015 , (b) 0.15 , (c) 1.5 and (d) $15 \mathrm{MeV}$. 

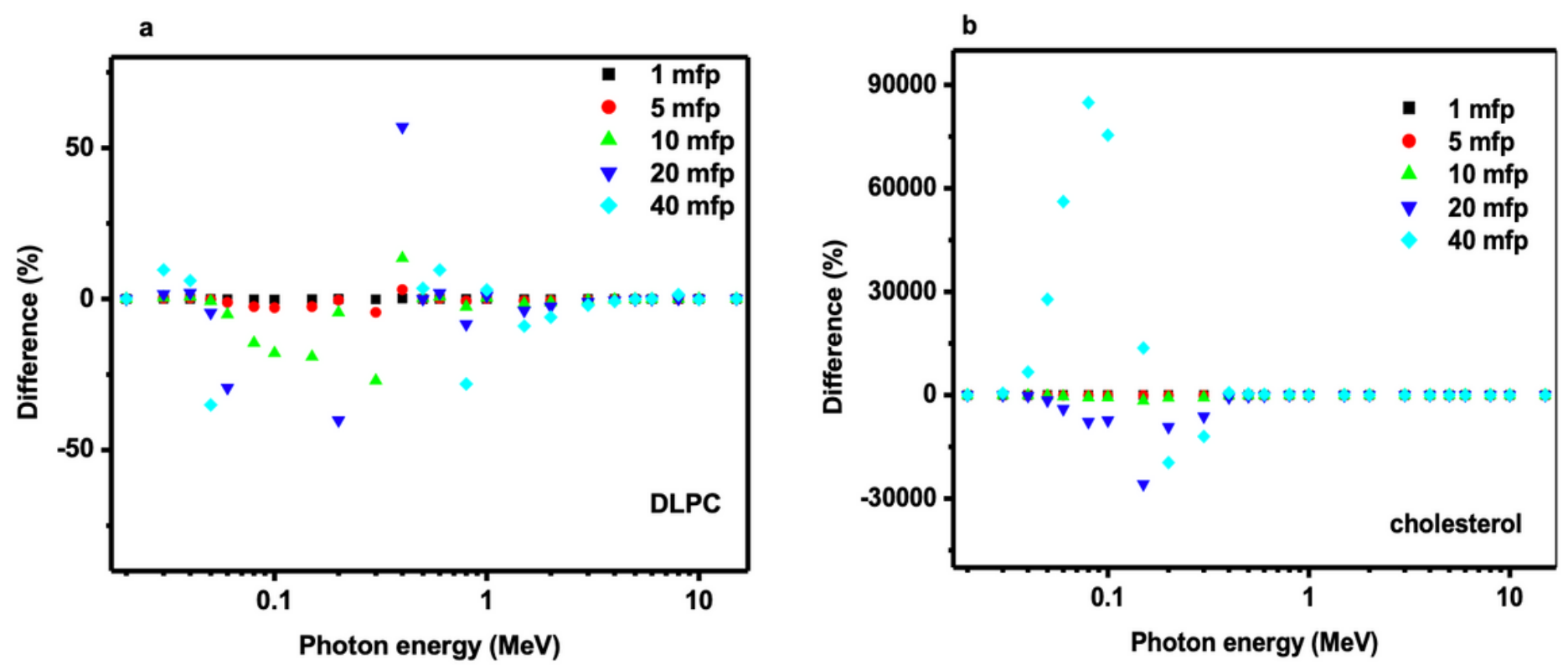

Figure 7

difference (\%) between EABF and EBF for (a) DLPC and (b) cholesterol in the energy region 0.015-15 MeV up to $40 \mathrm{mfp}$.

\section{Supplementary Files}

This is a list of supplementary files associated with this preprint. Click to download.

- Scheme210ctober2015v2.doc

- Supplmaterials3FebforGPmethod.doc 